\title{
A Novel Framework of Grid Cloud Operating and Comparison Analysis of its Virtualization Realization
}

\author{
Liang Zhang, Xiaoliang Bi \\ East-China Grid Company \\ Shanghai, China \\ zhang_liang@ec.sgcc.com.cn
}

\author{
Shudong Chen \\ Institute of Microelectronics of Chinese Academy of \\ Sciences, Beijing, China \\ chenshudong@ciotc.org
}

\begin{abstract}
In this paper, we propose a novel overall framework for Grid cloud operation based on cloud computing technologies. Considering the key role of virtualization software, we introduce three kinds of software which are popularly used in virtualization realization of the framework. From the point of view of CPU, memory and I/O, comparative tests have been done using different virtualization software and comparison analysis of the experimental results has been presented.
\end{abstract}

Keywords-cloud computing; grid cloud operation; virtualization; framework; comparison analysis

\section{INTRODUCTION}

As an important part of smart grid, intelligent grid operation is increasingly paid attention to recently. As we know, after many years of information system construction, almost Grid Operation Centers have been built up many information systems such as EMS(Energy Management System), SCADA(Supervisory Control And Data Acquisition), WARMS(Wide Area Measurement System), OPS(Operation schedule system), OMS(Operation Management System), electric energy acquisition and billing systems, electricity market operation system. Because these operation information systems are different to each other in hardware platforms, development languages, communication protocols, database structures, coupled with the security distribution and horizontal segregation of network in grid operation center for safety reasons, the problem of poor interaction and information integration between the these systems has appeared [1].

In order to solve this problem, a layered architecture of Cloud Operating is given in paper [2]. However, the paper [2] doesn't distinguish between global and local resources virtualization. On the other hand, it does not give the virtualization performance in detail. That is to say, paper [2] does not involve the following questions:

1) Management and coordination of local resources and global resources;

2) How to realize the virtual resource services?

3) What is the performance difference of virtualization softwares which are used to realize the resource allocation?

These problems will be discussed in this paper. Our contributions include a novel architecture of grid cloud operating which can meet demands of integration with the existing hardware resources, basic platforms, the established applications.

As we know, virtualization is the core and foundation of cloud operation framework. The performance problem of virtualization is related to the availability and reliability of the whole cloud operation. Therefore, the comparison analysis of the virtualization realization using several popular virtualization software is also discussed.

In the rest of the paper, we first overview related concept in grid cloud operating in Section 2. Then we propose a new framework of Grid Cloud Operating with detailed descriptions of the architecture (Section 3). In Section 4 we introduce three kinds of software which is popularly used in virtualization realization of our framework and the comparison Analysis. And then we discuss the results. Finally, we draw conclusions.

\section{Cloud Operating OVERVIEW}

\section{A. Cloud Computing}

The cloud computing is a variety of wide-area Internet integration of heterogeneous computing resources to form an abstract, virtual and can be dynamically expanded pool of computing resources; and it may provide computing power, storage capacity, software platforms and applications software and other services through the Internet to users on demand[2].

Cloud computing, a large-scale distributed computing paradigm, is driven by economies of scale, in which a pool of abstracted, virtualized, dynamically-scalable, managed computing power, storage, platforms, and services are delivered on demand to external customers over the Internet[3].

Cloud computing has the following characteristics: $\square$ integration of heterogeneous computing resources, $\square$ incremental scalability, service-oriented virtualized.

\section{B. Virtualization Technology}

In computing, virtualization refers to the act of creating a virtual (rather than actual) version of something, including (but not limited to) a virtual computer hardware platform, 
operating system (OS), storage device, or computer network resources[4].

As a method of logically dividing the system resources, virtualization technology, provided by mainframe computers between different applications[4].

\section{Cloud Operating}

Cloud operating is a new dispatching model, which uses cloud computing technology to organize and integrate various resources (hardware resources, software resources, platform resources) of all levels grid operation centers, and encapsulated them in the form of services for grid operating users to provide on-demand operating services[2].

\section{State Grid Operation cloud}

Each grid control centers have their own separate resources such as hardware resources, storage resources, software resources, platform resources and service resources. Those independent resources can be integrated to form a local sub-cloud, and then to form a national Grid Operation Cloud through the network connection and layered architecture[2].

The composition architecture of State Grid operation cloud is shown in Figure 1.

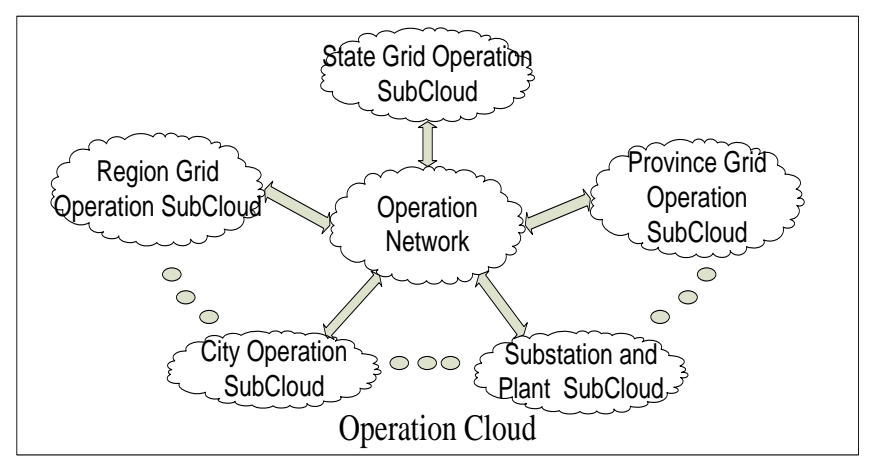

Fig. 1. The Composition Architecture of State Grid Operation Cloud

\section{NEW FRAMEWORK OF GRID CLOUd OPERATING}

The new layered framework of Grid Cloud Operation is shown in Figure 2. The framework is divided into five parts: Resource Cloud, Basic Platform Cloud, Application Cloud, Cloud Management and Cloud Interface.

1) Resource Cloud includes 3 layers: Physical Hardware Resource Layer, Local Virtual Resource Layer and Global Virtual Resource Layer. Physical Hardware Resource Layer includes the physical hardware resources such as CPU, memery and storage. Main role of Virtual Resource Layer is to map various types of physical hardware resources into virtual resources which is exceptionally easy organized and efficient. Virtual resources mainly include local and global virtual infrastructures, such as virtual storage, virtual network, virtual CPU.

2) Basic Platform Cloud, here especially refers to the D5000 basic platform cloud, includes Local Basic Platform Layer and Global Virtual Basic Platform Layer. Basic Platform involves workflow service, message service, GIS, rea-ltime information platform and so on. In order to solve the problem of poor interaction and information integration between the different systems, D5000 basic platform, an unified operation control system platform of smart grid, has been developed and deployed in the country.

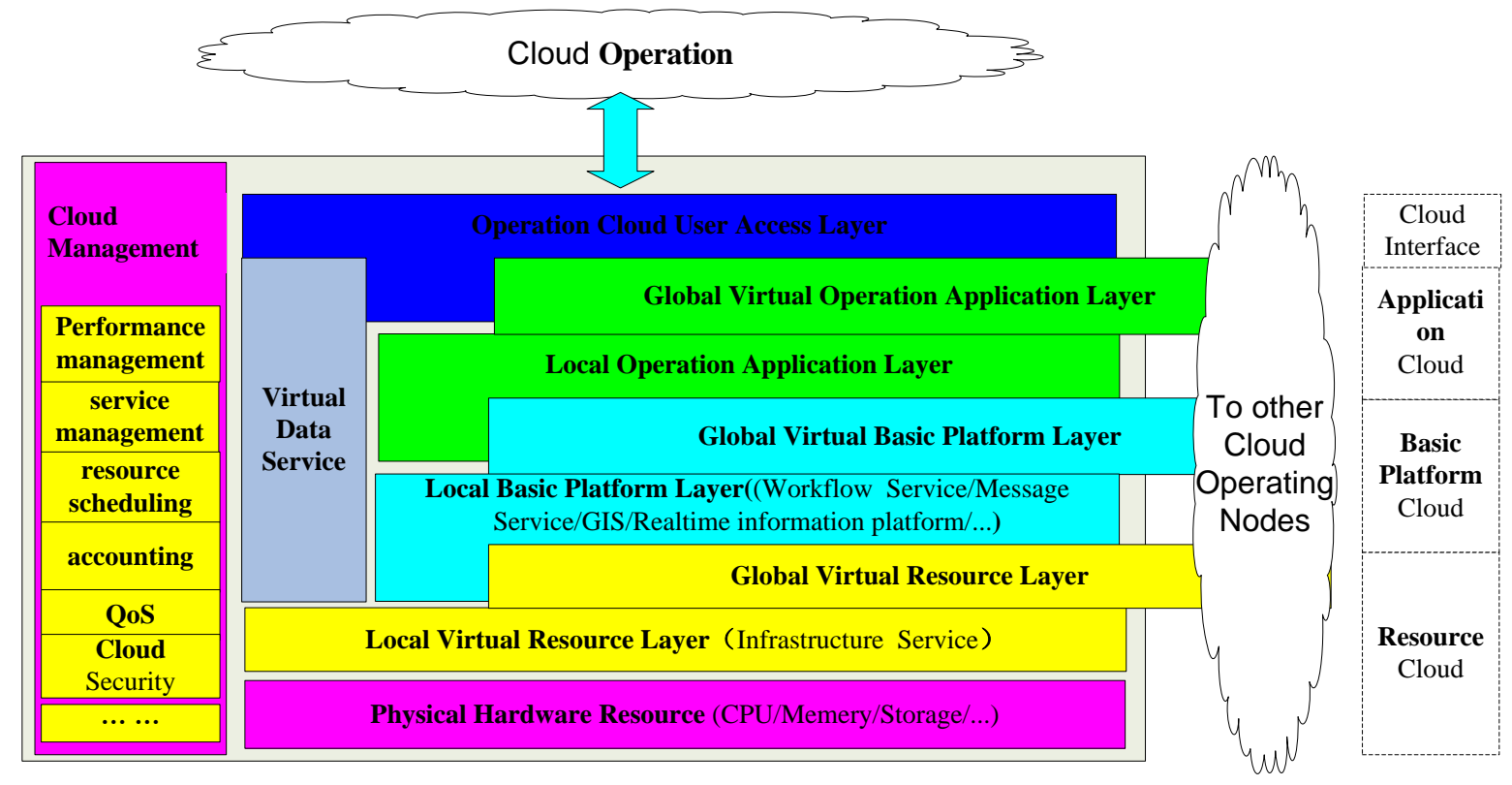

Fig. 2. The Cloud Computing-based layered architecture of national grid cloud 
3) Application Cloud includes Local Operation Application Layer and Global Virtual Operation Application Layer. Here application refers to business particles support service such as DPF(Dispatcher Power Flow), NS(Network Sensitivity), SE(State Estimation), PFC(Power Flow Calculation), SA(Security Analysis), VSA(Voltage Stability Analysis).Business Particles Support Service is to encapsulate certain business functions of virtual platform or application software systems into a service. For example, workflow platform services can make logical business integrations according to operation center's business demands, and then new business processes can be designed and implemented by calling the different services particles.

4) Operation Cloud User Access Layer: The user access ways of cloud operating have web applications, mobile device, java applications and web services gateway, $P C$, and so on. The Operation Cloud User access layer, which refers to the access that connects clouds to the users, provides unified interface to State Grid Cloud user.

5) Cloud Operation Management Layer, the vertical layer on the left side of Figure 2, represents additional technical support. Its specific functions includes performance management, access service, service management, QoS, resource scheduling management, accounting management, cloud security and so on. Security guarantees can be run through each layers, and are not a kind of technology for a specialized layer. For the loosely coupled distributed systems, security is particularly important. Operation cloud security provide basic security services functions for other services in the cloud architecture model, mainly including: authentication, access control, data encryption, data integrity and anti-denial, as well as security trust, identity, secure session, security strategies[2].

\section{EXPERIMENTAL COMPARISON}

As we know, virtualization is the core and foundation of cloud operation framework. The performance problem of virtualization is closely related to the availability and reliability of the whole cloud operation.

\section{A. The Experimental Environment}

The topological structure of the experiment system is shown in Figure 3.

Different kinds of virtualization software will be installed on the servers firstly, and then the Linux Rocky operating system software, DaMeng database software and D5000 platform. We choose three popular kinds of virtualization software: the VMware ESXi, Fusion compute and KVM in the test.

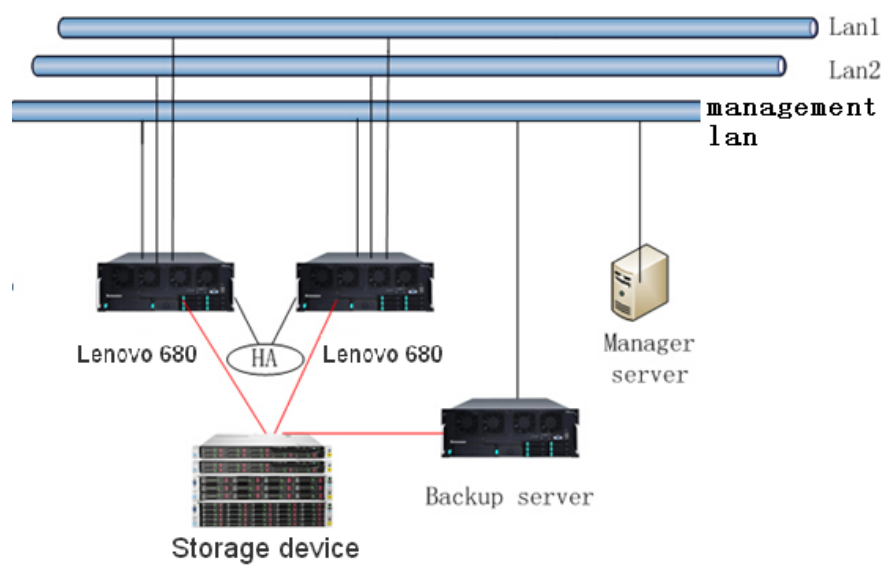

Fig. 3. The topological structure of the experiment system

\section{B. The Experimental Comparision}

From the three aspects of CPU, memory and I/O, several comparative tests have been conducted on different virtualization software.

\section{1) CPU Performance Testing}

UnixBench Tool provides a basic indicator of the performance of a Unix-like system. Multiple tests are used to test various aspects of the system's performance. These test results are then compared to the scores from a baseline system to produce an index value, which is generally easier to handle than the raw scores. The comparison analysis of CPU throughput performance is shown in Figure 4.

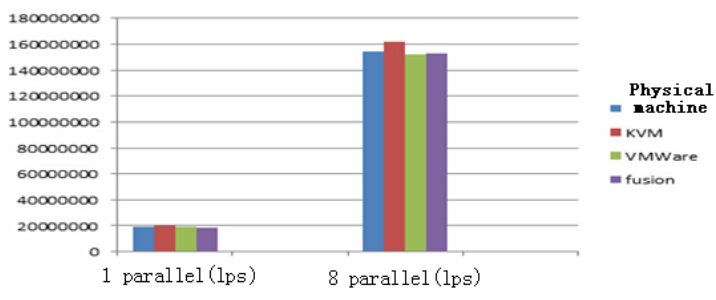

a) Dhrystone 2 using register variables

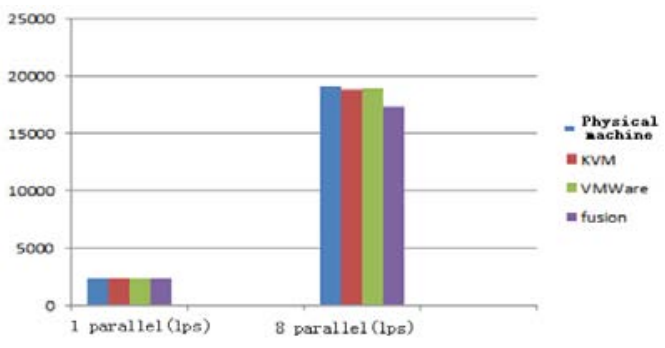

b) Double-Precision Whetstone 


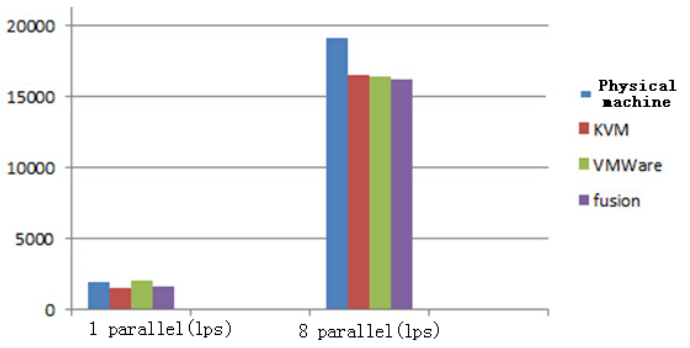

c) Execl Throughput

Fig. 4. Comparison Analysis of CPU throughput Performance

Figure 4 shows that ESXi, Fusion and KVM are almost the same in CPU throughput performance. KVM is slightly better than ESXi and Fusion.

TABLE I. COMPARISON OF THE FILE SYSTEM THROUGHPUT

\begin{tabular}{|l|c|c|c|c|}
\hline \multicolumn{1}{|c|}{ Type } & \multicolumn{2}{|c|}{ 1 parallel(KBps } & \multicolumn{2}{c|}{ 8 parallel(KBps } \\
\hline $\begin{array}{l}\text { Physical } \\
\text { machine }\end{array}$ & 611089.8 & $100 \%$ & 515284.6 & $100 \%$ \\
\hline KVM & 489884 & $84.77 \%$ & 489043.5 & $94.89 \%$ \\
\hline VMWare & 550303 & $95.74 \%$ & 526892 & $102.9 \%$ \\
\hline fusion & 514208.3 & $90.95 \%$ & 475693.8 & $93.01 \%$ \\
\hline
\end{tabular}

The file system throughput tests are used to test the data transfer rate from one file to another file. Different buffer sizes are used in each test. We statistics the numbers of file operations (read, write and copy) in given time (default is 10s). Table 1 gives the comparison data of the file system throughput.

\section{2) Memory Performance Testing}

The ram speed benchmark testing tool is used to test the Integer and Writing, Integer and Reading, Float and Writing, Float and Reading indicators in memory tests. The data in Figure 5 is the average value of the 4 indicators.

Block sizes began to grow from $1 \mathrm{~Kb}$ in the power of 2 , until the bounds of the array. The simple algorithm is used to measure the system cache and memory subsystems speed.

The comparison analysis of memory performance is shown in Figure 5.

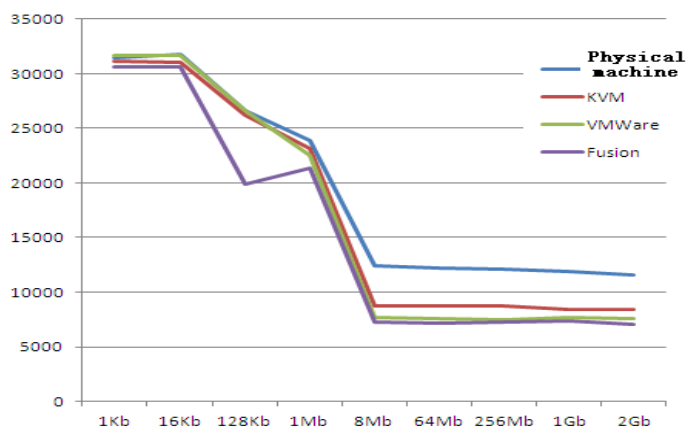

Fig. 5. Comparison Analysis of Memory Performance
From Fig. 5 we can see: when the data block is smaller than $128 \mathrm{~Kb}$, the memory performance of VMware ESXi is the best; when the data block is larger, KVM is stronger than the VMware and Fusion; Fusion is the worst in Memory Performance.

\section{3) Disk I/O Performance Testing}

The tool of iozone is used to test disk performance.

The comparison analysis of memory performance is shown in Figure 6.

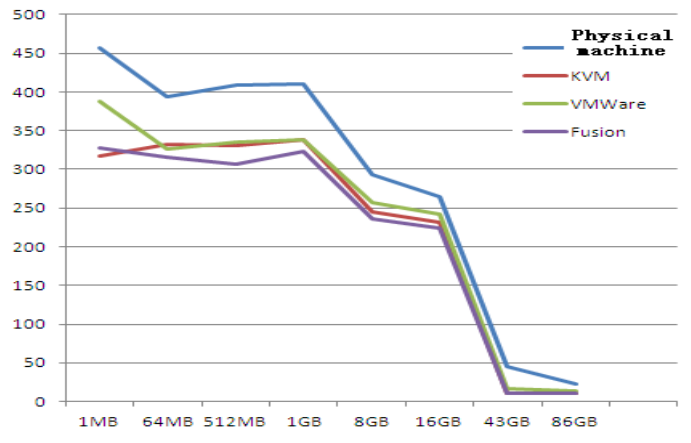

Fig. 6. Comparison Analysis of Disk I/O Performance

Fig.6 tells us that VMware ESXi is the best in disk I/O performance. Furthermore, read operation performance of $\mathrm{KVM}$ is strongest, and the write operation performance of VMware is the best.

\section{CONCLUSION}

Based on cloud computing technologies and combined with East China Grid Operation Center IT architecture, this paper proposed a new Cloud Operation overall framework. And then, we introduce three kinds of software adopted for the virtualization realization of our framework. Finally, experimental tests were carried out using different virtualization software and comparison analysis of the test results on CPU, memory and I/O was presented.

\section{References}

[1] Liang ZHANG, Minhui Ge, Xiaoliang Bi, “A SOA-BPM-Based Architecture for Intelligent Power Dispatching System”, Power and Energy Engineering Conference (APPEEC), 2010 Asia-Pacific.

[2] Liang ZHANG, Lei ZHANG, and Minhui, et al, "Reaserch and Application on the Cloud-Computing-Based Power Dispatching IT Architecture”, Power and Energy Engineering Conference (APPEEC), 2011 Asia-Pacific.

[3] FOSTER I , ZHAO Y, RAICU I, “Cloud computing and grid computing 360 degree compared”, Proceedings of Grid Computing Environment s Workshop , November 12-16, 2008, Austin , TX, USA: 1-10.

[4] http://en.wikipedia.org/wiki/ 